\title{
BMJ Open Healthcare utilisation in overweight and obese children: a systematic review and meta-analysis
}

\author{
Taimoor Hasan (DD , , ${ }^{1,2}$ Tom S Ainscough, ${ }^{1}$ Jane West (D) , ${ }^{2}$ \\ Lorna Katharine Fraser (D) ${ }^{1}$
}

To cite: Hasan T, Ainscough TS, West J, et al. Healthcare utilisation in overweight and obese children: a systematic review and meta-analysis. BMJ Open 2020;10:e035676. doi:10.1136/ bmjopen-2019-035676

- Prepublication history for this paper is available online. To view these files, please visit the journal online (http://dx.doi. org/10.1136/bmjopen-2019035676).

Received 14 November 2019 Revised 21 June 2020 Accepted 14 August 2020

Check for updates

(c) Author(s) (or their employer(s)) 2020. Re-use permitted under CC BY-NC. No commercial re-use. See rights and permissions. Published by BMJ.

${ }^{1}$ Department of Health Sciences, University of York, York, UK

${ }^{2}$ Bradford Institute for Health Research, Bradford Teaching Hospitals NHS Foundation Trust, Bradford, UK

Correspondence to

Dr Taimoor Hasan;

th1132@york.ac.uk

\section{ABSTRACT}

Objective This systematic review and meta-analysis aims to systematically analyse the association of overweight and obesity with health service utilisation during childhood. Data sources PubMed, MEDLINE, CINAHL, EMBASE and Web of Science.

Methods Observational studies published up to May 2020 that assessed the impact of overweight and obesity on healthcare utilisation in children and adolescents were included. Studies were eligible for inclusion if the included participants were $\leq 19$ years of age. Findings from all included studies were summarised narratively. In addition, rate ratios (RRs) and 95\% Cls were calculated in a metaanalysis on a subgroup of eligible studies.

Outcome measures Included studies reported association of weight status with healthcare utilisation measures of outpatient visits, emergency department (ED) visits, general practitioner visits, hospital admissions and hospital length of stay.

Results Thirty-three studies were included in the review. When synthesising the findings from all studies narratively, obesity and overweight were found to be positively associated with increased healthcare utilisation in children for all the outcome measures. Six studies reported sufficient data to meta-analyse association of weight with outpatient visits. Five studies were included in a separate meta-analysis for the outcome measure of ED visits. In comparison with normal-weight children, rates of $\mathrm{ED}$ (RR $1.34,95 \% \mathrm{Cl} 1.07$ to 1.68) and outpatient visits (RR $1.11,95 \% \mathrm{Cl} 1.02$ to 1.20 ) were significantly higher in obese children. The rates of ED and outpatient visits by overweight children were only slightly higher and nonsignificant compared with normal-weight children. Conclusions Obesity in children is associated with increased healthcare utilisation. Future research should assess the impact of ethnicity and obesity-associated health conditions on increased healthcare utilisation in children with overweight and obesity.

PROSPERO registration number CRD42018091752

\section{INTRODUCTION}

In recent years, childhood obesity has emerged as one of the greatest paediatric public health concerns worldwide. According to latest report by WHO, in 2016 over 41 million children under the age of 5 , and over 340 million children and adolescents

\section{Strengths and limitations of this study}

- A systematic search of the published literature in English language in major databases up to May 2020 was conducted.

- Risk of bias was assessed in the included studies and the review is reported according to Preferred Reporting Items for Systematic reviews and MetaAnalysis guidelines.

- Search of grey literature, unpublished studies and studies published in a language other than English was not conducted.

Meta-regression analysis could not be conducted.

aged 5-19, were overweight or obese globally. ${ }^{1}$ The situation is of serious concern in the UK, which is reported to be the most obese country in Western Europe by the Organisation of Economic Co-operation and Development. ${ }^{2}$ Recent reports have shown that 1 in 5 children in the reception year (age 4-5) and 1 in 3 children in year 6 (age 12-13) are obese or overweight in the UK. ${ }^{3}$

The burden of obesity-related morbidity is well documented. Extensive research has shown that individuals who are obese or overweight in their childhood are more likely to stay overweight or obese in adult life, ${ }^{4}$ leading to an increased risk of developing cardiometabolic conditions such as type 2 diabetes, ischaemic heart disease and stroke. ${ }^{4-6}$ In addition, the increasing prevalence of overweight and obesity in childhood has led to an increase in the incidence of previously unusual metabolic imbalances at this age, and a rise in associated diseases such as type 2 diabetes and metabolic syndrome..$^{7-11}$

Given the aforementioned associations, it could be inferred that individuals with overweight and obesity would experience greater morbidity compared with individuals of normal weight, leading to increased healthcare utilisation. Several studies have reported a strong association between overweight 
or obesity and increased healthcare use. ${ }^{12-14}$ However, majority of these have quantified this association by assuming that individuals with obesity will start accruing the obesity-associated increased healthcare use at or after a certain age, with most ignoring the healthcare use during childhood. ${ }^{12} 15$

In order to address this issue, we conducted a systematic review and meta-analysis with the objective of evaluating the association of overweight or obesity with healthcare utilisation in children, pooling the available evidence from eligible studies. In this review, we also aim to identify the obesityassociated conditions that may explain the association of overweight or obesity with increased healthcare utilisation.

\section{METHODS}

This review is reported in accordance with the Preferred Reporting Items for Systematic reviews and Meta-Analysis (PRISMA) recommendations. ${ }^{16}$ The protocol for this review is registered with PROSPERO-International Prospective Register of Systematic Reviews. The PRISMA checklist is provided as online supplemental file 1 .

\section{Literature search}

A systematic literature search was performed in five electronic databases (PubMed, Medline, EMBASE, Web of Science and CINAHL) from inception to July 2018. An update of database searches was conducted in May 2020. This search update covered the full data range from inception to May 2020, and records found in the previous search were removed based on the methods described by Bramer and Bain. ${ }^{17}$ The search focused on studies reporting association between weight status and healthcare utilisation in children. Only studies published in English language were considered for inclusion. The searches were conducted by assembling terms that could relate to the three main components of the review: 'children or adolescents', 'obesity or overweight' and 'healthcare utilisation'. These terms comprised keywords, text terms or medical subject headings appropriate for each literature database. A copy of the searches conducted to identify studies is given in online supplemental file 2. We also searched the reference lists of screened publications to look for additional articles. A forward and backward reference search for all the studies meeting the inclusion criteria was carried out to identify any other relevant studies. Research reported in grey literature was not searched. Conference abstracts and review articles were not eligible for inclusion. However, reference lists of screened review articles were checked for potentially relevant studies.

\section{Study eligibility}

Observational studies assessing the impact of overweight or obesity on healthcare utilisation in children were included in the review. Studies were excluded based on the following criteria: studied the association for underweight children only; included participants over 19years of age; included participants both less than and greater than 19years of age but did not stratify the results by age groups; review articles.
The decision for the inclusion of children/adolescents up to the age of 19years was made based on WHO's definition of a child and adolescent. ${ }^{18}$ In addition, instead of restricting the inclusion criteria to studies using predefined standard body mass index (BMI) cut-offs for childhood overweight (sex-specific and age-specific BMI $\geq 85$ th centile and $<95$ th centile) and obesity (sex-specific and age-specific BMI $\geq 95$ th centile), ${ }^{1920}$ a decision was made to include the study-specific definitions with the aim of assessing the effect of varying BMI cut-offs on the association of overweight or obesity with healthcare utilisation.

\section{Study selection}

Titles and abstracts of records retrieved through literature search up to July 2018 were screened by a single reviewer (TH) with a random sample of $10 \%$ of these studies screened by a second reviewer (TSA). Studies were then full text screened by the first reviewer (TH) to assess their eligibility for inclusion in the review. A random sample of $10 \%$ of these full-text studies was also screened by the second reviewer (TSA). The level of agreement between the two reviewers at each stage was assessed by Cohen's kappa score. The score was classified as follows: $<0.20$ indicated a poor agreement; $0.21-0.40 \mathrm{a}$ fair agreement; $0.41-0.60$ a moderate agreement; $0.61-0.80$ a good agreement; $0.81-1.00$ a very good agreement. ${ }^{21}$ All disagreements were resolved through discussion between the two reviewers and by consulting a third reviewer (LKF) if required.

Additional records retrieved from the search update in May 2020 were screened for title, abstract and full text by the first reviewer $(\mathrm{TH})$.

\section{Data extraction and risk of bias assessment}

A customised data extraction form was designed to extract following information from each study: first author's surname, year of publication, study design, country, sample size, age range, time frame, definition of obesity/overweight, outcome measures and effect size for healthcare use. Data for each study were extracted by the first reviewer $(\mathrm{TH})$ and reviewed by the second reviewer (TSA). Any discrepancies were discussed and resolved through consensus between the reviewers.

The Quality Assessment tool for Observational Cohort and Cross-sectional studies by the National Heart and Lung Institute (NHLBI) was used to assess the quality and risk of bias of each included study. ${ }^{22}$ This assessment tool rates study quality along 14 criteria, with three possible outcomes for each question: 'Yes', 'No' and 'Cannot determine/Not reported/Not applicable'. For a response of 'Yes', a score of one was assigned against the criteria, whereas a score of zero was assigned for any answer other than 'Yes'. Each study was then rated Good, Fair or Poor based on a score ranging from 0 to 14 ; where a 'good' study was considered to have the least risk of bias, 'fair' was susceptible to some bias and 'poor' indicated a high risk of bias.

\section{Narrative synthesis}

Due to the diverse nature of healthcare utilisation outcomes, measures of effect and lack of appropriate or sufficient data 
in the majority of studies to statistically analyse these effect size measurements, a decision was made to summarise the findings of the included studies narratively. A narrative synthesis was developed to explain the impact of weight status on all the reported measures of health service use in different studies: emergency department visits, outpatient visits, general practitioner (GP) visits, hospital admissions and length of stay (LOS). In addition, potential sources of heterogeneity across studies were explored.

\section{Statistical analysis}

The 'meta' command in Stata V.16.1 ${ }^{23}$ was used to generate meta-analysis for rate ratios (RRs) of healthcare utilisation in obese and overweight children, using normal-weight children as a reference. Studies that reported RRs with corresponding measures of precision (95\% CIs or SEs) were included in the meta-analysis. In addition, studies with appropriate raw data to compute crude RRs were eligible for inclusion in the meta-analysis. Meta-analysis uses effect sizes in a metric that makes them closest to normally distributed; therefore, before undertaking the analysis in Stata, RRs were log transformed and corresponding SEs were computed from effect sizes and $95 \%$ CIs using the Comprehensive Meta-Analysis software V.3. ${ }^{24}$ Afterwards, a random-effects meta-analysis with Hartung-Knapp-Sidik-Jonkman method was carried out. ${ }^{25} 26$ The error rates for this method have consistently been shown to be more robust than the more commonly used DerSimonian and Laird method, particularly when there are small number of studies in the meta-analysis. ${ }^{27}$

Publication bias was assessed using funnel plots; however, due to the number of studies included in the analysis being less than 10 , statistical tests for funnel plot asymmetry were not performed. ${ }^{28}$ Heterogeneity among studies was assessed using the $\mathrm{I}^{2}$ statistic. Based on the interpretation provided in the Cochrane Handbook for Systematic Reviews, heterogeneity in this review is considered substantial if $\mathrm{I}^{2}>50 \% .^{29}$

\section{Patient and public involvement}

No patients or members of public were involved in the conduct and reporting of this review.

\section{RESULTS}

\section{Study selection}

A PRISMA flow diagram for study selection is shown in figure 1. The search of electronic databases up to July 2018 identified 36077 records. After removal of duplicates, 18966 studies were screened by titles and abstracts. A random sample of 1900 studies $(10 \%)$ was also reviewed by the second reviewer. The level of agreement between reviewers at this stage was reflected by a Cohen's kappa

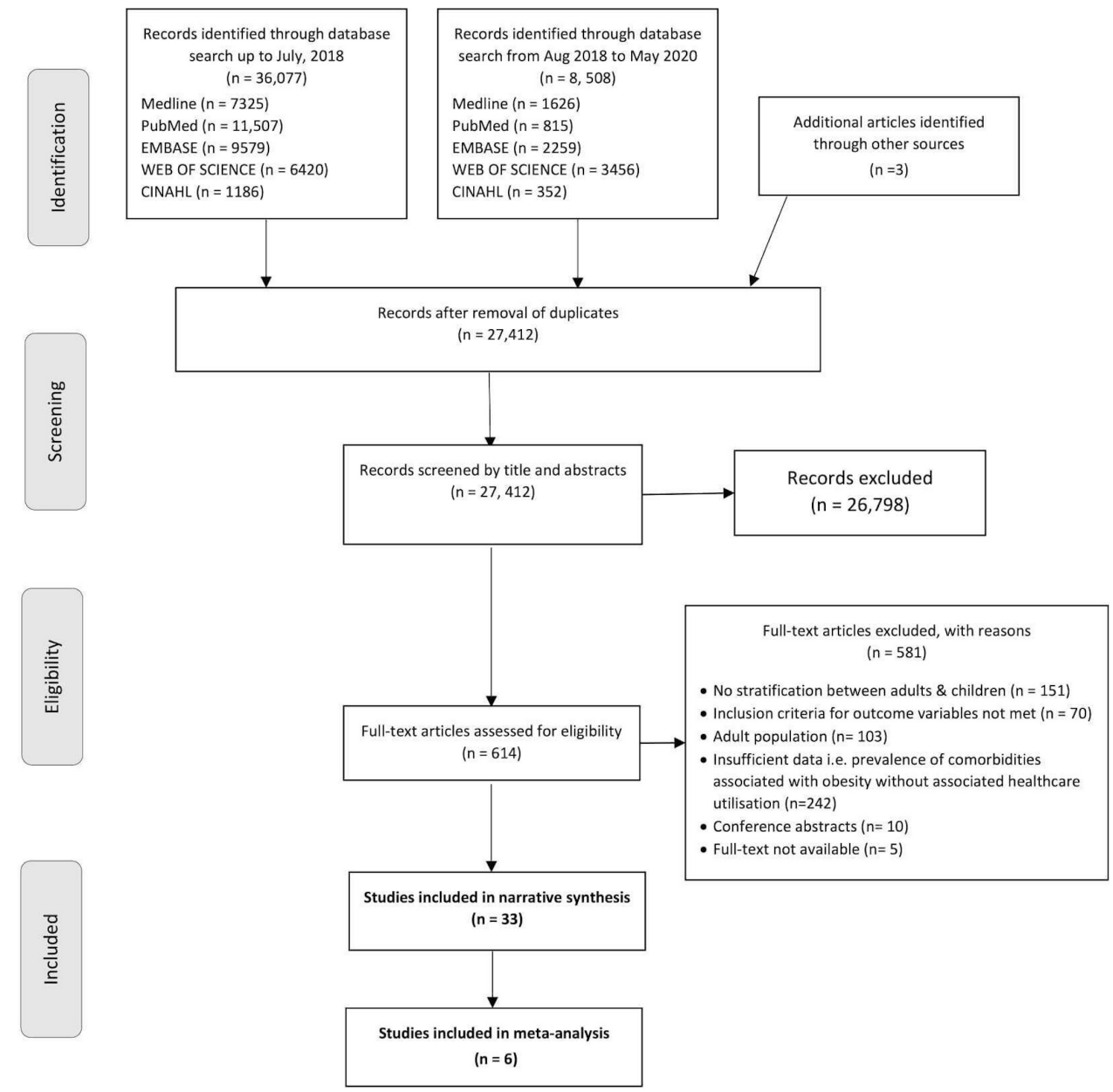

Figure 1 Preferred Reporting Items for Systematic Reviews and Meta-Analyses (PRISMA) study selection diagram. 
score of 0.86 . Full texts of 578 studies were screened by the first reviewer with a random sample of 60 studies $(10 \%)$ also reviewed by the second reviewer. Cohen's kappa score for level of agreement at this stage was 0.67 , which indicated a good agreement. Twenty-six articles were eligible for inclusion at this stage.

The search update in May 2020 identified 8504 additional articles, of which 4 were eligible for inclusion. Three additional articles were identified through searching the reference lists of screened systematic reviews. Overall, 33 studies were eligible for inclusion. All these studies were included in the narrative synthesis, but only six were included in the meta-analysis.

\section{Study characteristics}

The basic characteristics of included studies are summarised in table 1 . The majority of these studies $(n=20)$ were conducted in the USA. Twenty-three of the included studies were cohort studies. Nine of the remaining studies used crosssectional methods, while one study was a case-control study (table 1). Multiple studies reported data from two surveys/ cohorts. The Medical Expenditure Panel Survey (MEPS) is reported in five studies ${ }^{30-34}$ and the German Interview and Examination Survey for Children and Adolescents (KiGGS) is reported in two studies. ${ }^{35}{ }^{36}$ As studies from the same survey/cohort reported data for different years or different outcome measures, decision was made to analyse the data for each individual study.

Table 1 summarises the measures of healthcare utilisation reported across the included studies. The most commonly reported outcome measures were emergency department (ED) visits $(\mathrm{n}=10)^{32-34} 37-43$ and outpatient $(\mathrm{n}=11)$ visits (including primary care and specialty visits). ${ }^{32-34} 36-414344$ Seven studies reported on healthcare use associated with respiratory diseases, ${ }^{414-49}$ two reported on musculoskeletal conditions ${ }^{440}$ and two on conditions concerning mental health. ${ }^{3744}$ The rest of the studies analysed the overall healthcare use in children with no reporting on reasons for utilisation. The studies represented children between 1 and 19years of age. Table 1 shows that seven studies calculated BMI from anthropometric measurements (height and weight) based on selfreported or parent-reported data. ${ }^{30-3451} 52$ In all other studies, heights and weights were either measured as part of the study or recorded from the health facility records. Two studies reported data on weight only and used weight:age ratio to define obesity or overweight. ${ }^{5354}$ In addition, different variables were adjusted for in the multivariate analysis in respective studies. These variables are listed in table 1 .

\section{Risk of bias}

The response for each study against the criteria in NHLBI's quality assessment tool to critically appraise the internal validity is shown in table 2 . Fourteen studies scored a 'good' rating, sixteen had a 'fair' rating, while three had a 'poor' rating. The studies included in the meta-analysis were either of 'good' or 'fair' quality; therefore, weighting based on quality assessment was not done in the meta-analysis. However, quality assessment was used to weigh the strength of evidence during narrative synthesis.

\section{Narrative synthesis and meta-analysis}

Findings from all included studies were synthesised narratively for each outcome measure of healthcare utilisation. A subgroup synthesis was done by dividing studies based on BMI cut-offs, ethnicity and method of anthropometric measurement.

Six studies were included in the meta-analysis. ${ }^{373840414355}$ All of these studies were cohort studies (table 1). All six studies reported an association between weight status and outpatient visits and were included in the meta-analysis for outcome measure of outpatient visits. Five of these six studies also reported on association of weight status with ED visits, and were therefore included in a separate metaanalysis for outcome measure of ED visits. ${ }^{37} 38404143 \mathrm{In}$ addition, five of these $\mathrm{s}^{373841455}$ used a similar definition to define obesity (age-specific and sex-specific BMI $\geq 95$ th percentile) while one study ${ }^{40}$ defined it as age-specific and sex-specific BMI z-score $\geq 2$, which also corresponds to BMI $\geq 95$ th percentile. ${ }^{19}$ Moreover, five studies included in the meta-analysis for ED visits were conducted in the USA. The sixth study, which was part of analysis for outpatient visits, was conducted in Canada. For one study, ${ }^{38}$ the appropriate effect sizes with corresponding SEs were calculated using the available raw data. One study assessed healthcare use over 1-year and 3-year periods. A decision was made to include data for 1-year period due to larger sample size as many participants were lost to follow-up by the end of the 3-year period. ${ }^{37}$ Figures 2 and 3 show the forest plots for meta-analysis with outcome measures of ED visits and outpatients visits, respectively. Online supplemental figures 1 and 2 show forest plots for ED and outpatient visits in obese children compared with normal-weight children calculated using the pre-specified adjusted RRs reported by individual studies. Due to a small number of studies eligible for inclusion in the metaanalysis and limited to no data available on key covariates, it was not possible to perform a subgroup analysis.

\section{ED visits}

Ten studies reported ED visits as an outcome measure for healthcare utilisation. ${ }^{32-34} 37-414353$ In both obese and overweight children compared with normal-weight children, the general direction of association was an increase in visits; however, variability in the strength and direction of association was reported. For obese children compared with normal-weight children, five studies reported a significant increase in ED visits. ${ }^{32} 33404143$ Three studies reported a non-significant increase in ED visits. ${ }^{37-39} \mathrm{In}$ addition, one study reported a non-significant decrease of ED visits in obese children 6-11 years old, while for obese children aged 12-17 years, a significant increase in visits was reported. ${ }^{34}$ For overweight children, four studies reported a significant increase in ED visits compared with normal-weight children. ${ }^{32} 334143$ Two studies reported a 


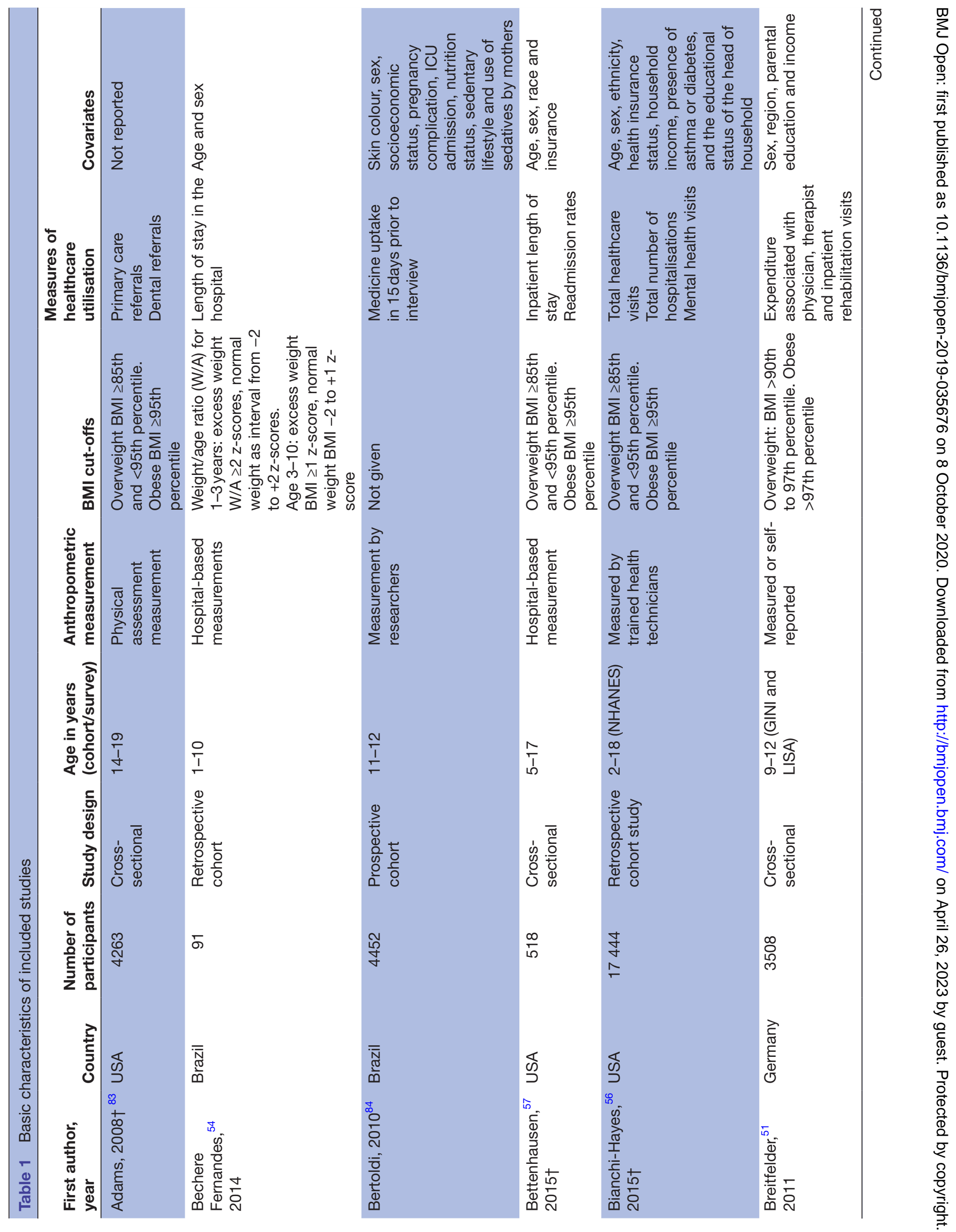




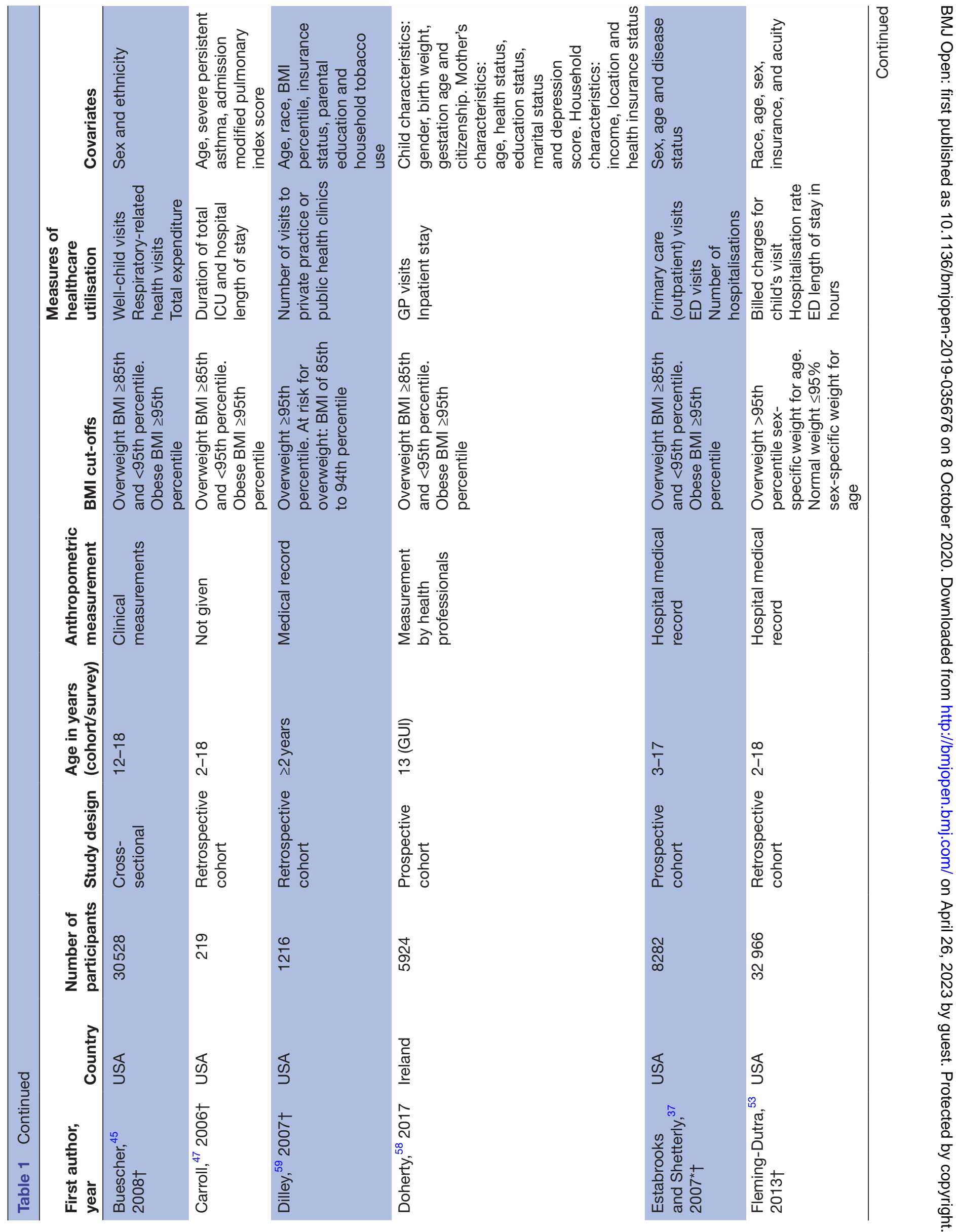









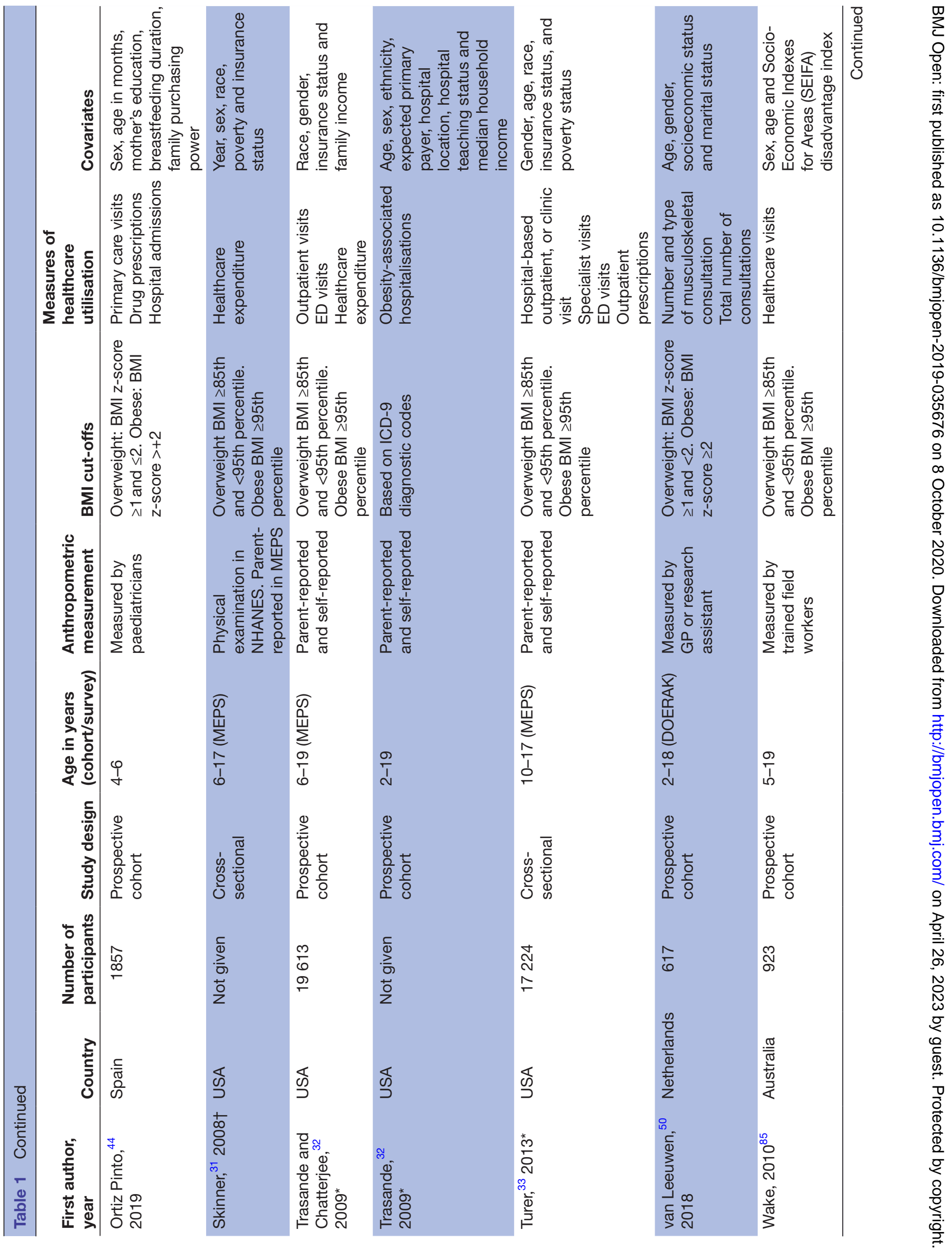




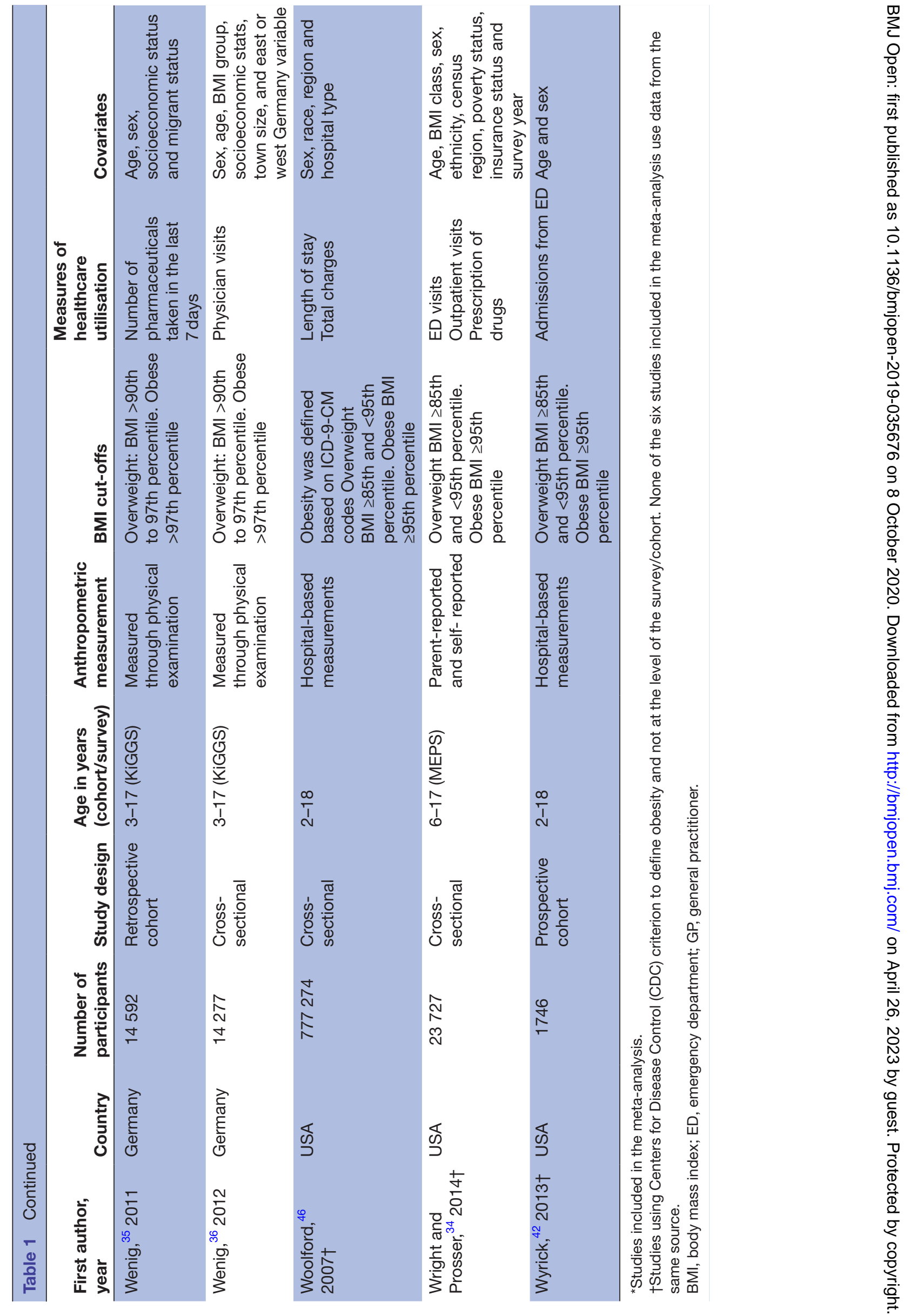




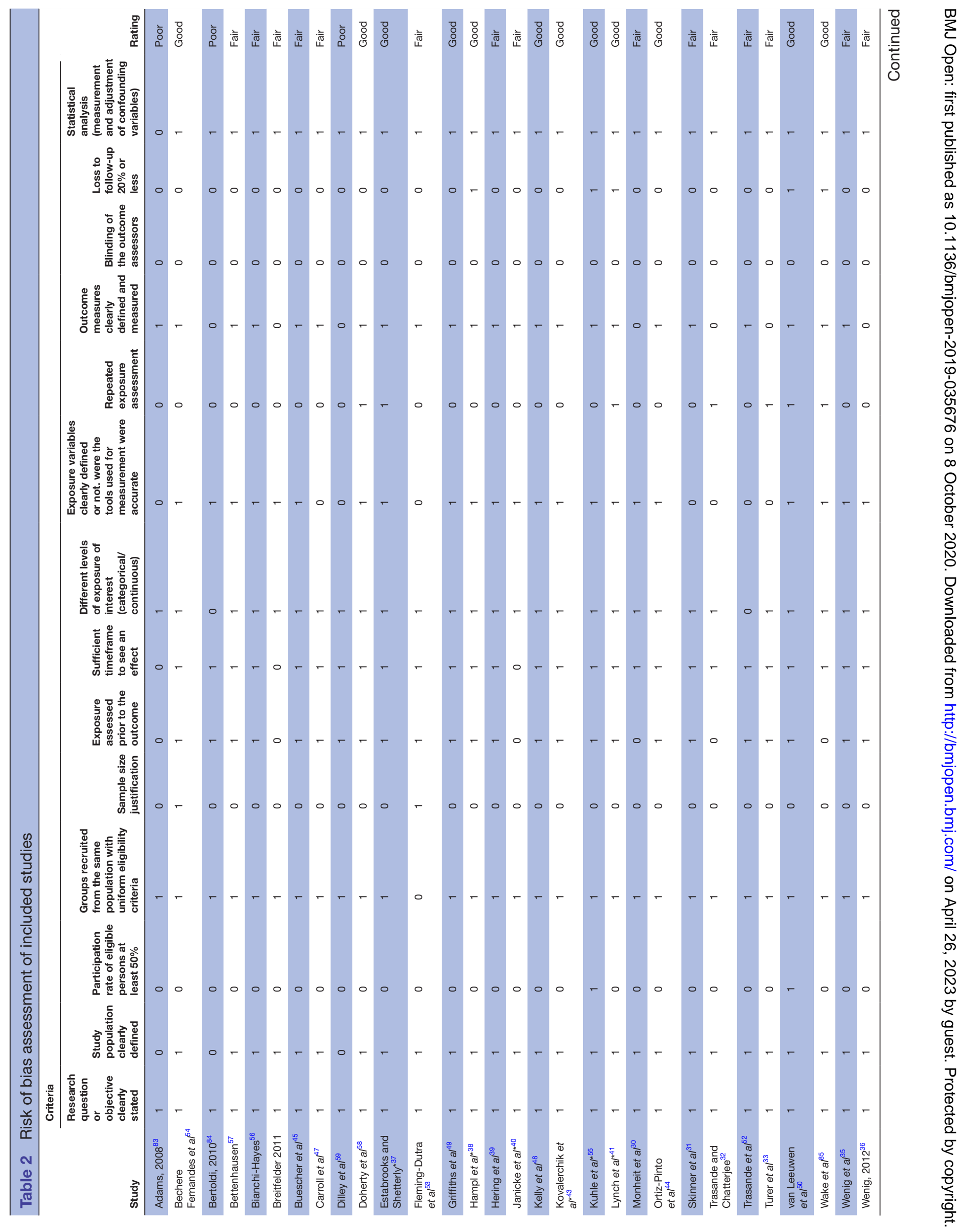




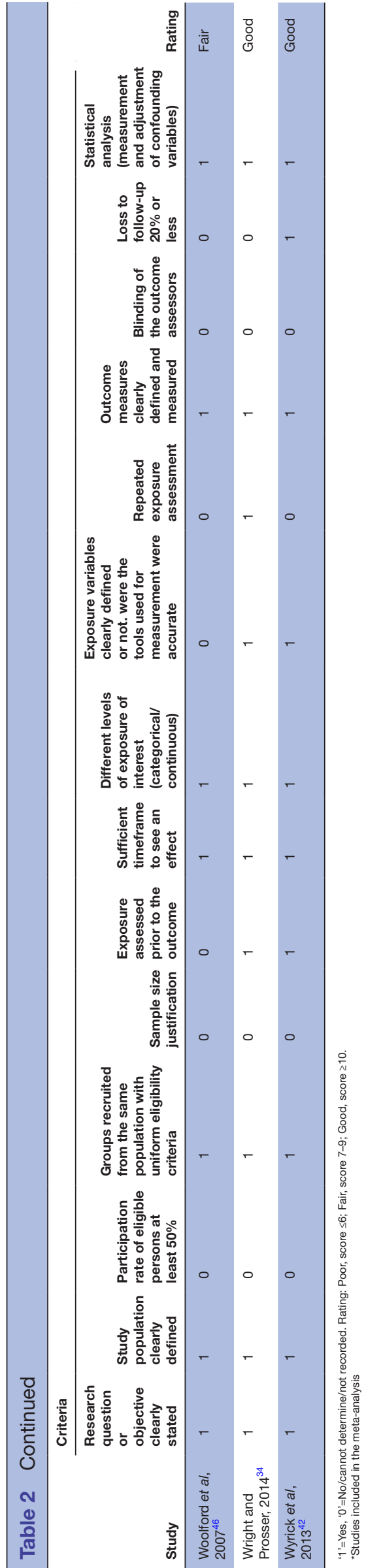

non-significant increase ${ }^{34} 38$ and two studies reported a non-significant decrease. ${ }^{3740}$

In the five studies included in the meta-analysis for ED visits, obese children were significantly more likely to visit EDs compared with normal-weight children (figure 2A). The associated effect size (RR) was 1.34 (95\% CI 1.07 to 1.68). The effect size for overweight versus healthy weight was RR 1.11 (95\% CI 0.92 to 1.33 ) (figure 2B). The I ${ }^{2}$ statistic showed substantial between-study heterogeneity for obese versus normal weight $\left(\mathrm{I}^{2}=94.3 \%, \mathrm{p}<0.01\right)$ and overweight versus normal weight $\left(\mathrm{I}^{2}=92.5 \%, \mathrm{p}<0.01\right)$.

On visual inspection of funnel plot asymmetry, there is a possibility of publication bias, with a small sized study reporting high RRs for obese children (online supplemental figure 3). A statistical test for publication bias was not performed due to small number of studies $(\mathrm{n}<10)$.

\section{Outpatient visits}

Eleven studies reported outpatient visits as a measure of healthcare utilisation. $.^{32-34} 36-414344$ In obese children compared with normal-weight children, the general direction of association was an increase in visits; however, variability in the strength of association was reported. Seven studies reported a significant increase in outpatient visits for obese children, ${ }^{32333739-4143}$ while four studies reported a non-significant increase. ${ }^{34363844}$ For overweight children compared with normal-weight children, three studies reported a significant increase in outpatient visits. ${ }^{37} 4143$ Five studies reported a non-significant increase ${ }^{32-34} 3638$ while two studies reported a non-significant decrease in outpatient visits. ${ }^{4044}$

Pooled unadjusted RRs for obese versus normal weight and overweight versus normal weight were 1.11 (95\% CI 1.02 to 1.20 ) and 1.02 (95\% CI 0.98 to 1.08 ), respectively (figure 3A,B). Significant between-study heterogeneity was observed for both obese versus normal-weight children $\left(\mathrm{I}^{2}=87.6 \%, \mathrm{p}<0.01\right)$ and overweight versus normalweight children $\left(I^{2}=73 \%, p<0.01\right)$.

Visual inspection of funnel plot asymmetry for outpatient visits in obese children suggests publication bias (online supplemental figure 4). Statistical tests to assess publication bias were not performed due to the small number of studies $(\mathrm{n}<10)$.

\section{Hospital admissions and LOS}

Seven studies reported hospital admissions as a measure of healthcare use. ${ }^{37} 394142444956$ One study reported a significant increase ${ }^{39}$ while two studies reported a nonsignificant increase ${ }^{37} 49$ in hospital admissions for obese children compared with normal weight. Two studies reported a non-significant decrease in admissions. ${ }^{44}{ }^{56} \mathrm{In}$ addition, one study reported that $14.5 \%$ of obese or overweight children were admitted, compared with $16.5 \%$ normal-weight children. ${ }^{42}$ For overweight children, one study reported a significant decrease ${ }^{56}$ while one reported a non-significant decrease ${ }^{37}$ in admissions compared with normal-weight children. 
A

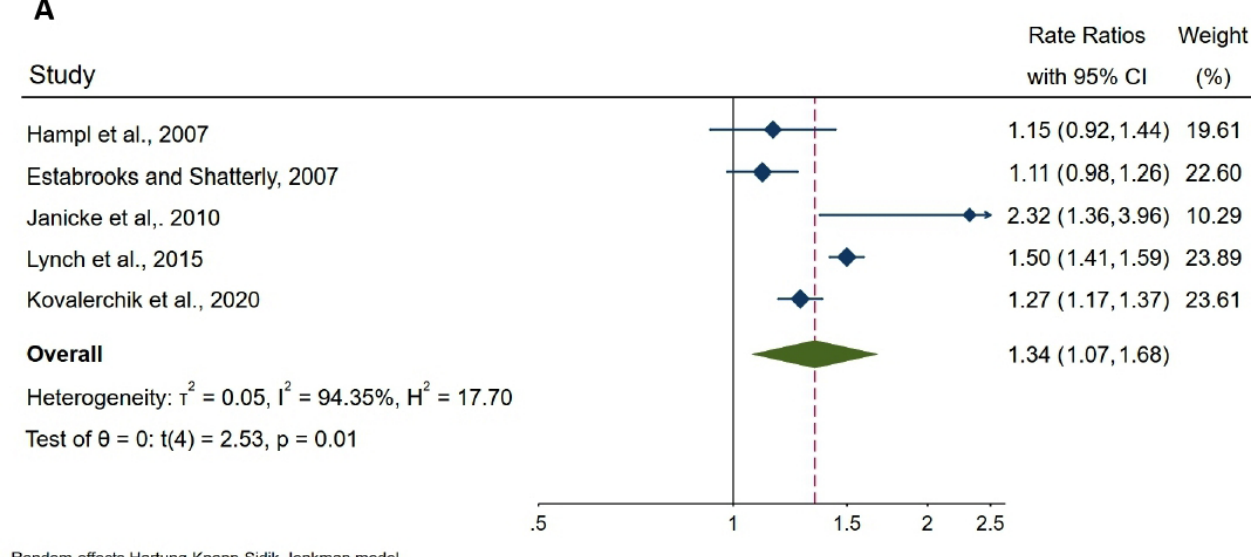

Random-effects Hartung-Knapp-Sidik-Jonkman model

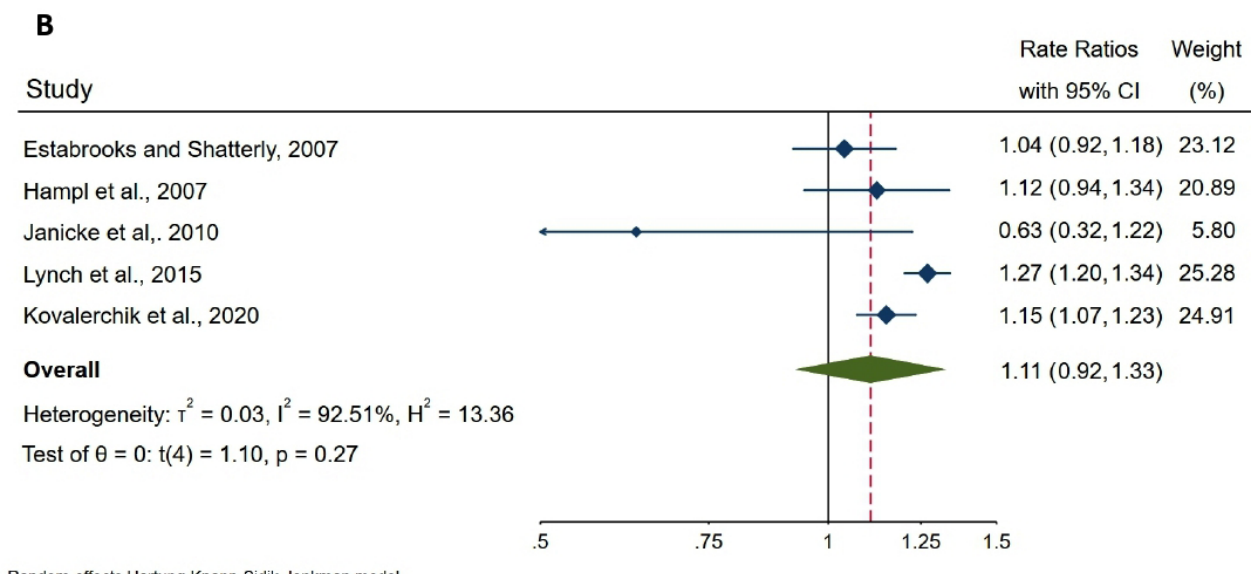

Figure 2 Forest plots showing the unadjusted effect sizes (rate ratios (RRs) with 95\% Cls) for emergency department visits in (A) obese children, (B) overweight children. RRs are computed with normal-weight children as the reference category.

Hospital LOS was reported as a measure of healthcare utilisation by six studies. ${ }^{46} 4752-5457$ Four studies found a significant increase in LOS for obese children compared with normal weight. ${ }^{4645254}$ One study reported a slight significant decrease in LOS for obese children, ${ }^{57}$ while one study reported no association between obese and normal-weight children. ${ }^{53}$

\section{GP visits}

Three studies reported GP visits as a measure for healthcare utilisation. ${ }^{48558}$ All three studies reported a significant increase in GP visits for overweight and obese children, compared with their normal-weight peers.

\section{Associated medical conditions}

Five studies reported on the effect of asthma or acute respiratory disorders on healthcare utilisation in obese children. ${ }^{41}{ }^{45-48}$ Of these studies, four reported that obese children significantly incurred increased healthcare use for asthma compared with normal-weight children. ${ }^{45-48}$ In addition, two studies found that other acute respiratory conditions are also significantly associated with increased healthcare use in obese children. ${ }^{41}{ }^{45}$ Furthermore, two studies reported a non-significant increase for respiratory conditions in obese children. ${ }^{44} 46$
Two studies reported that obese children are at a significantly greater risk of seeking healthcare for mental health problems compared with normal-weight children. ${ }^{37} 44$ The risk for overweight children was also reported to be higher but non-significant. Two studies reported a nonsignificant increase in visits for musculoskeletal problems in obese children compared with normal-weight children. ${ }^{44} 50$

\section{BMI cut-offs}

Table 1 shows that 20 of the included studies used the Centers for Disease Control or the International Obesity task force cut-off points to classify children into weight categories. However, some studies used the term 'overweight' in place of obese for $\geq 95 \%$ percentile, while using the term 'at-risk of overweight (AROW)' in place of overweight for children with BMI percentiles $\geq 85 \%$ and $\leq 95 \%$. During the analysis, we adjusted for this difference in terminologies.

Two studies used the weight for age BMI z-score classification. ${ }^{40} 54$ The effect size reported by these two studies for obese children was significant and much stronger than the studies not using this criterion. Three studies using data from German survey KiGGs and GINI and 
A

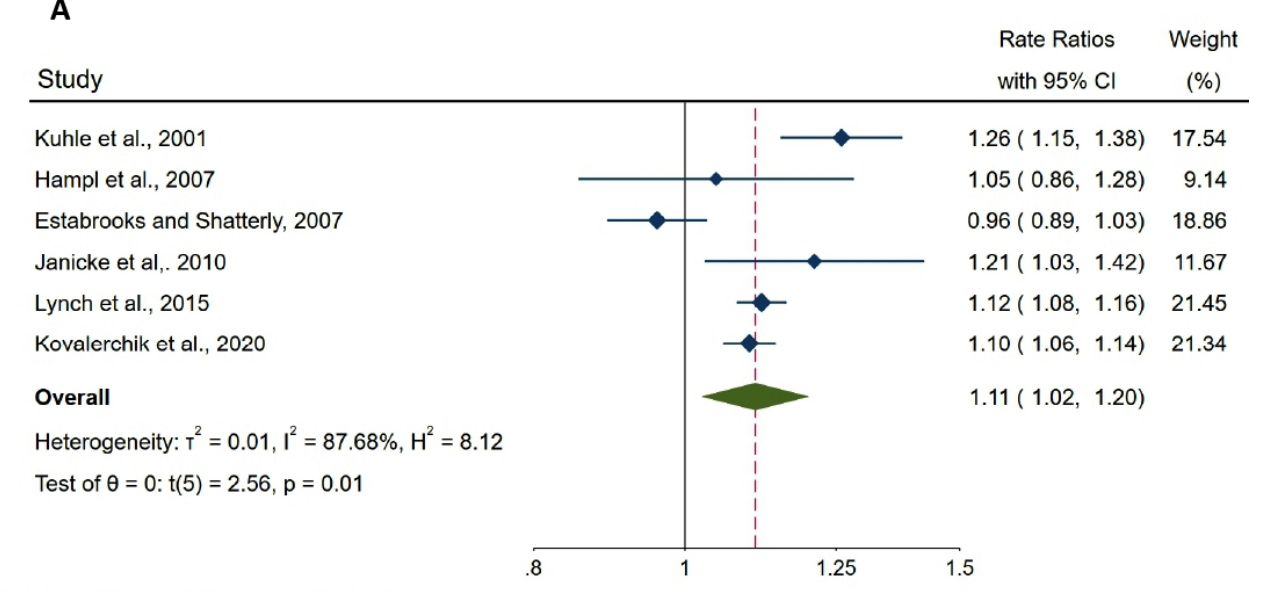

Random-effects Hartung-Knapp-Sidik-Jonkman model

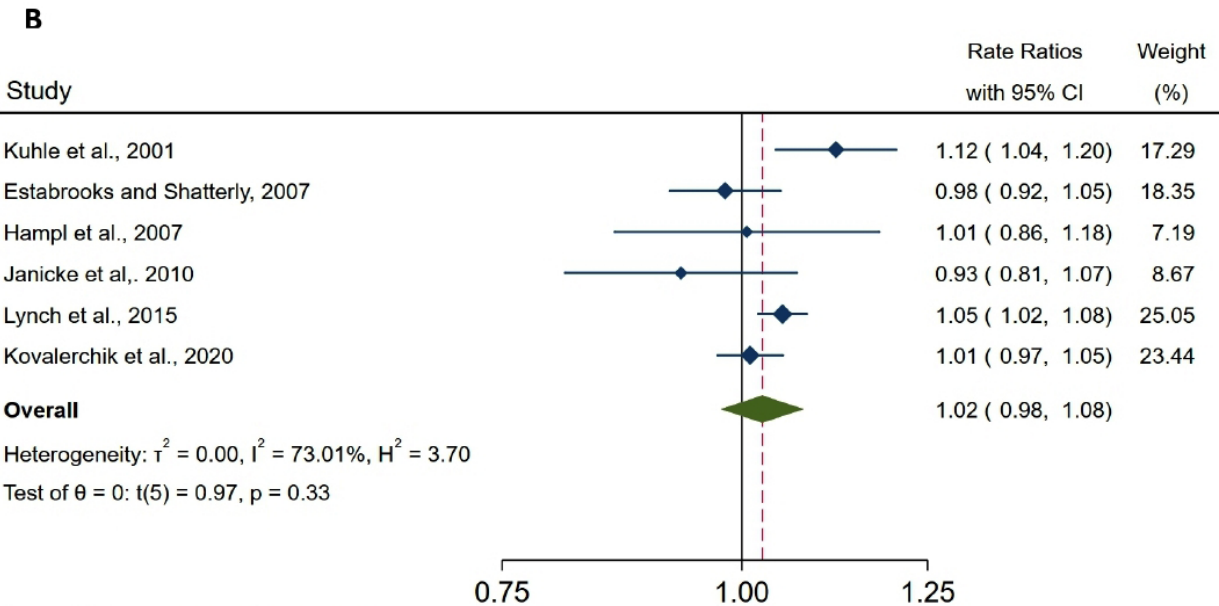

Random-effects Hartung-Knapp-Sidik-Jonkman model

Figure 3 Forest plots showing the unadjusted effect sizes (rate ratios (RRs) with 95\% Cls) for outpatient visits in (A) obese children, (B) overweight children. RRs are computed with normal-weight children as a reference category.

LISA cohorts used the country-specific BMI cut-off values with obesity defined as $>97$ th percentile. ${ }^{353651}$ It was not possible to formally establish a comparison based on BMI cut-off criteria due to the small number of studies using respective BMI cut-offs and the use of different outcome measures across these studies.

\section{Ethnicity}

Two studies reported the effect of ethnicity on the association of weight status with healthcare utilisation. ${ }^{30} 59$ Both these studies were from the USA. They reported a decrease in healthcare utilisation in black overweight or obese children compared with white overweight or obese children. In addition, one study also reported decreased healthcare use in obese Asian or Hispanic children compared with white obese children. ${ }^{30}$

\section{Anthropometric measurements}

Seven studies recorded the height and weight by self-reporting or parental reporting without validation. ${ }^{30-345152}$ Five of these studies used data from MEPS survey in the USA. Variability in the direction and strength of association between weight status and healthcare use was observed across these studies. This heterogeneity could be subject to reporting bias due to self-reporting or parent-reporting; however, not enough data were available to formally assess this.

\section{DISCUSSION}

This systematic review and meta-analysis has demonstrated an association between excess weight and increased healthcare use in children. Thirty-three studies were included in the review, of which six had appropriate data to be included in the meta-analysis. Attesting to the diverse nature of health services and the variability in their provision in different countries, the studies used multiple outcome measures to define healthcare utilisation. Commonly examined outcome measures were outpatient visit, ED visit, hospital admission and hospital LOS. Studies included in the meta-analysis reported an increased risk of healthcare utilisation in obese children compared with normal-weight children. A significant 
unadjusted positive association of obesity with increased outpatient and ED visits was observed in the meta-analysis. The results of the narrative synthesis supported these findings and indicated that obese children are much more likely to have higher healthcare utilisation for all the reported outcome measures. However, variability in the direction and strength of association was observed across studies, with a few studies reporting a negative or no association.

A vast body of research and associated systematic reviews exist which have analysed the burden of adult obesity on healthcare systems and also the incremental health burden of child obesity during adulthood. ${ }^{60-62}$ Such studies have indicated repeatedly that obesity is significantly related to a greater risk of morbidity in adult life and associated increase in healthcare utilisation. Our review builds on this knowledge and suggests that much like adult life, obesity during childhood results in an increased burden of morbidity on healthcare services. These findings can be explained in the light of recent clinical research reporting an increasing prevalence of obesity-related conditions in childhood that were more commonly associated with adulthood in the past. ${ }^{763}$

This leads our discussion into one of the secondary objectives of the review: to analyse the most common obesity-associated health conditions that are contributing to an increased healthcare use in children with obesity. Most of the included studies did not attempt to ascertain the reason for increased healthcare utilisation. Two studies included in the review analysed the rate of mental health related visits in obese children, with both reporting an increased risk. These findings support the previous evidence that has shown obesity to be a strong risk factor for stigmatisation and development of low self-esteem and other mental health issues in children. ${ }^{64}{ }^{65}$ The role of obesity in increasing the risk of asthma in children is well founded. ${ }^{66}$ Five studies in the review supported the previous evidence and reported that obesity leads to increased health service utilisation in asthmatic children and also in children with other respiratory diseases.

Regional variation in rates of healthcare utilisation is well reported in literature ${ }^{67-69}$ When studies conducted in different regions or countries with different population characteristics and healthcare systems are systematically reviewed and analysed together, regional variation in healthcare utilisation may result in between-study heterogeneity. Evidence suggests that this regional variation is in part driven by population-specific factors such as ethnicity, socioeconomic status, health status, cultural beliefs and preferences. ${ }^{68}$ The prevalence of childhood obesity varies between different regions and countries. It is also well reported that within a population, the prevalence of obesity varies between children of different ethnic origins. ${ }^{3} 7071$ In addition, evidence shows an inverse relationship between the prevalence of obesity and low socioeconomic status. ${ }^{3}{ }^{72}$ The extent to which this variability in prevalence translates into variability in associated morbidity and healthcare use is not known.
There is evidence that healthcare seeking behaviour and healthcare uptake varies across ethnic groups and socioeconomic classes. ${ }^{73-76}$ Most of this evidence suggest that people belonging to black and other minority ethnic groups are at a disadvantage in accessing health services. ${ }^{77} 78$ In addition, cultural beliefs and perceptions towards health status in general and weight status in particular may contribute to ethnic disparities in healthcare utilisation. ${ }^{79}{ }^{80}$ None of the studies included in the review analysed the impact of socioeconomic status while only two studies analysed the impact of ethnicity. They reported a significantly lower use of health services in obese children of black, Asian and other ethnic minority groups compared with white children. To what extent this lower use is a result of disadvantage in access to healthcare and what results from differences in prevalence and in levels of morbidity remains unclear. In addition, both of these studies were from the USA, which has specific health insurance programmes for children. ${ }^{81} 82$ Therefore, care should be taken in generalising these findings to other countries with different healthcare systems. In the light of these two studies and previous research evidence, we can infer that ethnicity and socioeconomic status could be sources of between-study heterogeneity reported in this review; however, as the studies did not report the ethnic and socioeconomic characteristics of the populations studied, it was not possible to explore this further. Evidence also suggests that in addition to populationspecific factors, regional variation in healthcare is in part due to differences in region-specific factors such as access to health services, healthcare resources, health policies and physician beliefs. ${ }^{68}{ }^{69}$ For example, some percentage of the between-study heterogeneity reported in our review may be attributable to regional variations in physician beliefs towards excess weight or barriers and facilitators to healthcare access. However, exploring the extent of heterogeneity due to region-specific variables was beyond the scope of this review.

\section{Strengths and limitations}

This review has a number of strengths. First, to our knowledge this is the first systematic review and meta-analysis of the utilisation of healthcare services in obese and overweight children. Second, we have used a comprehensive search strategy, with publications not restricted by region or year of publication which resulted in the inclusion of 33 studies reporting outcome measures from primary and secondary healthcare. In addition, a protocol was developed and registered a priori and methodological guidelines were followed on conducting and reporting a review.

A limitation of this review was the restriction of studies to English-language reports only. A limitation of the metaanalysis was the inclusion of only six studies which meant we were unable to include all the outcomes described in the review. In addition, there was uncertainty over the weighted effect sizes due to between-study heterogeneity in methods and outcomes. 
There were some further limitations in terms of the characteristics of the included studies. First, the majority of the studies were from the USA, with the remainder being from eight first-world countries, therefore limiting the extent to which the findings may be generalised beyond certain national contexts due to differences in healthcare services and systems. Second, there was poor reporting of data for key study characteristics. For example, none of the studies included in the meta-analysis reported the use of healthcare services stratified by sex. Therefore, it was not possible to run a subset analysis and adjust for covariates in a meta-regression to formally analyse sources of between-study heterogeneity.

\section{CONCLUSIONS}

In summary, this systematic review has shown that overweight and obesity in children is positively associated with increased utilisation of ED and outpatient healthcare services during childhood. This finding remained in the meta-analysis although with potential heterogeneity between studies. The reported evidence for inpatient health service use is mixed. The studies included in the review are limited to only a few developed countries; therefore, it is difficult to generalise these findings to other countries due to differences in healthcare systems and delivery of health services. The substantial betweenstudy heterogeneity reported in the review might be due to these differences across countries; however, it was not possible to formally analyse this due to insufficient data. The review has identified areas of research where gaps exist. In particular, further research is required in understanding the dynamics of obesity-associated health conditions that may drive increased healthcare utilisation in children. In addition, the driving factors behind the varying effect of ethnicities and socioeconomic status on association of obesity with healthcare utilisation are yet to be explored. Such evidence is necessary for the development of policies for clinical practice and research, and for their implementation in a way that, while being costeffective, can successfully target the therapeutic needs of obese and overweight children from different ethnic and socioeconomic backgrounds.

\section{Twitter Taimoor Hasan @TaimoorHasan13}

Contributors TH conceptualised and designed the protocol, conducted the literature search and screening, assessed risk of bias, extracted data, conducted the narrative synthesis and meta-analysis, drafted the initial manuscript and revised the manuscript. TSA screened the studies, reviewed the extraction of data and quality assessment, and revised the initial and subsequent drafts. JW and LKF designed the protocol, revised the initial and subsequent manuscript drafts and approved the final version for publication. All authors approved the final manuscript as submitted and agree to be accountable for all aspects of work.

Funding The authors have not declared a specific grant for this research from any funding agency in the public, commercial or not-for-profit sectors.

Competing interests None declared.

Patient consent for publication Not required.

Provenance and peer review Not commissioned; externally peer reviewed.

Data availability statement № additional data are available.
Open access This is an open access article distributed in accordance with the Creative Commons Attribution Non Commercial (CC BY-NC 4.0) license, which permits others to distribute, remix, adapt, build upon this work non-commercially, and license their derivative works on different terms, provided the original work is properly cited, appropriate credit is given, any changes made indicated, and the use is non-commercial. See: http://creativecommons.org/licenses/by-nc/4.0/.

\section{ORCID iDs}

Taimoor Hasan http://orcid.org/0000-0003-3607-3490

Jane West http://orcid.org/0000-0002-5770-8363

Lorna Katharine Fraser http://orcid.org/0000-0002-1360-4191

\section{REFERENCES}

1 Organization WH. Taking action on childhood obesity. World Health Organization, 2018.

2 OECD. Health at a glance 2017, 2017.

3 NHS Digital. Statistics on Obesity, Physical Activity and DietEngland, 2018, 2018.

4 Singh AS, Mulder C, Twisk JWR, et al. Tracking of childhood overweight into adulthood: a systematic review of the literature. Obes Rev 2008;9:474-88.

5 Bray GA, Kim KK, Wilding JPH, et al. Obesity: a chronic relapsing progressive disease process. A position statement of the World Obesity Federation. Obes Rev 2017;18:715-23.

6 Llewellyn A, Simmonds M, Owen CG, et al. Childhood obesity as a predictor of morbidity in adulthood: a systematic review and metaanalysis. Obes Rev 2016;17:56-67.

7 Abbasi A, Juszczyk D, van Jaarsveld CHM, et al. Body mass index and incident type 1 and type 2 diabetes in children and young adults: a retrospective cohort study. J Endocr Soc 2017;1:524-37.

8 Lang JE, Obesity LJE. Obesity, nutrition, and asthma in children. Pediatr Allergy Immunol Pulmonol 2012;25:64-75.

9 Chen F, Wang Y, Shan X, et al. Association between childhood obesity and metabolic syndrome: evidence from a large sample of Chinese children and adolescents. PLoS One 2012;7:e47380.

10 Vukovic R, Zdravkovic D, Mitrovic K, et al. Metabolic syndrome in obese children and adolescents in Serbia: prevalence and risk factors. J Pediatr Endocrinol Metab 2015;28:903-9.

11 Daniels SR, Arnett DK, Eckel RH, et al. Overweight in children and adolescents: pathophysiology, consequences, prevention, and treatment. Circulation 2005;111:1999-2012.

12 Finkelstein EA, Graham WCK, Malhotra R. Lifetime direct medical costs of childhood obesity. Pediatrics 2014;133:854-62.

13 Kent S, Green J, Reeves G, et al. Hospital costs in relation to bodymass index in 1.1 million women in England: a prospective cohort study. Lancet Public Health 2017;2:e214-22.

14 Dee A, Kearns K, O'Neill C, et al. The direct and indirect costs of both overweight and obesity: a systematic review. BMC Res Notes 2014;7:242

15 Tucker DMD, Palmer AJ, Valentine WJ, et al. Counting the costs of overweight and obesity: modeling clinical and cost outcomes. Curr Med Res Opin 2006;22:575-86.

16 Moher D, Liberati A, Tetzlaff J, et al. Preferred reporting items for systematic reviews and meta-analyses: the PRISMA statement. Ann Intern Med 2009;151:264-9.

17 Bramer W, Bain P, PJJotMLAJ B. Updating search strategies for systematic reviews using EndNote. JMLA 2017;105.

18 World Health Organization. Definition of key terms, 2013. Available: http://www.who.int/hiv/pub/guidelines/arv2013/intro/keyterms/en/

19 Dinsdale H, Ridler C, Ells L. A simple guide to classifying body mass index in children. Oxford, UK: National Obesity Observatory, 2011.

20 Centers for Disease Control and Prevention. Defining Childhood Obesity | Overweight \& Obesity | CDC, 2019. Available: https://www. cdc.gov/obesity/childhood/defining.html

21 Altman DG. Practical statistics for medical research. CRC Press, 1990.

22 National Heart L, Institute B. Quality assessment tool for observational cohort and cross-sectional studies. Bethesda: National Institutes of Health, Department of Health and Human Services, 2014.

23 StataCorp. StataCorp LJCS, Stata. release 16.0. Texas: StataCorp LP, 2019.

24 Borenstein M, Hedges L, Higgins J, et al. Comprehensive metaanalysis. 2013. Version 3. NJ, 2013.

25 Hartung J, Knapp G. A refined method for the meta-analysis of controlled clinical trials with binary outcome. Stat Med 
26 Sidik K, Jonkman JN, Analysis D. Robust variance estimation for random effects meta-analysis. Comput Stat Data Anal 2006;50:3681-701.

27 IntHout J, loannidis JPA, Borm GF, GFJBmrm B. The HartungKnapp-Sidik-Jonkman method for random effects meta-analysis is straightforward and considerably outperforms the standard DerSimonian-Laird method. BMC Med Res Methodol 2014;14:25.

28 Sterne JAC, Sutton AJ, loannidis JPA, et al. Recommendations for examining and interpreting funnel plot asymmetry in meta-analyses of randomised controlled trials. BMJ 2011;343:d4002.

29 Higgins J, Green S. Cochrane handbook for systematic reviews of interventions. version 5.1. 0. The Cochrane Collaboration, 2011.

30 Monheit AC, Vistnes JP, Rogowski JA. Overweight in adolescents: implications for health expenditures. Econ Hum Biol 2009;7:55-63.

31 Skinner AC, Mayer ML, Flower K, et al. Health status and health care expenditures in a nationally representative sample: how do overweight and healthy-weight children compare? Pediatrics 2008;121:e269-77.

32 Trasande L, Chatterjee S. The impact of obesity on health service utilization and costs in childhood. Obesity 2009;17:1749-54.

33 Turer CB, Lin H, Flores G. Health status, emotional/behavioral problems, health care use, and expenditures in overweight/obese us children/adolescents. Acad Pediatr 2013;13:251-8.

34 Wright DR, Prosser LA. The impact of overweight and obesity on pediatric medical expenditures. Appl Health Econ Health Policy 2014;12:139-50.

35 Wenig CM, Knopf $\mathrm{H}$, Menn P. Juvenile obesity and its association with utilisation and costs of pharmaceuticals - results from the KiGGS study. BMC Health Serv Res 2011;11:340.

36 Wenig CM. The impact of BMI on direct costs in children and adolescents: empirical findings for the German healthcare system based on the KiGGS-study. Eur J Health Econ 2012;13:39-50.

37 Estabrooks PA, Shetterly S. The prevalence and health care use of overweight children in an integrated health care system. Arch Pediatr Adolesc Med 2007;161:222-7.

38 Hampl SE, Carroll CA, Simon SD, et al. Resource utilization and expenditures for overweight and obese children. Arch Pediatr Adolesc Med 2007;161:11-14.

39 Hering E, Pritsker I, Gonchar L, et al. Obesity in children is associated with increased health care use. Clin Pediatr 2009;48:812-8.

40 Janicke DM, Harman JS, Jamoom EW, et al. The relationship among child weight status, psychosocial functioning, and pediatric health care expenditures in a Medicaid population. $J$ Pediatr Psychol 2010;35:883-91.

41 Lynch BA, Finney Rutten LJ, Jacobson RM, et al. Health care utilization by body mass index in a pediatric population. Acad Pediatr 2015;15:644-50.

42 Wyrick S, Hester C, Sparkman A, et al. What role does body mass index play in hospital admission rates from the pediatric emergency department? Pediatr Emerg Care 2013;29:974-8.

43 Kovalerchik O, Powers E, Holland ML, et al. Differences in frequency of visits to pediatric primary care practices and emergency departments by body mass index. Acad Pediatr 2020;20:532-9.

44 Ortiz-Pinto MA, Ortiz-Marrón H, Esteban-Vasallo MD, et al. Demand for health services and drug prescriptions among overweight or obese preschool children. Arch Dis Child 2020;105:292-7.

45 Buescher PA, Whitmire JT, Plescia M. Relationship between body mass index and medical care expenditures for North Carolina adolescents enrolled in Medicaid in 2004. Prev Chronic Dis 2008;5:A04.

46 Woolford SJ, Gebremariam A, Clark SJ, et al. Incremental hospital charges associated with obesity as a secondary diagnosis in children. Obesity 2007;15:1895-901.

47 Carroll CL, Bhandari A, Zucker AR, et al. Childhood obesity increases duration of therapy during severe asthma exacerbations. Pediatr Crit Care Med 2006;7:527-31.

48 Kelly B, West J, Yang TC, et al. The association between body mass index, primary healthcare use and morbidity in early childhood: findings from the Born In Bradford cohort study. Public Health 2019;167:21-7.

49 Griffiths LJ, Cortina-Borja M, Bandyopadhyay A, et al. Are children with clinical obesity at increased risk of inpatient hospital admissions? An analysis using linked electronic health records in the UK millennium cohort study. Pediatr Obes 2019;14:e12505.

50 van Leeuwen J, van Middelkoop M, Paulis WD, et al. Overweight and obese children do not consult their general practitioner more often than normal weight children for musculoskeletal complaints during a 2-year follow-up. Arch Dis Child 2018;103:149-54.

51 Breitfelder A, Wenig CM, Wolfenstetter SB, et al. Relative weightrelated costs of healthcare use by children-results from the two
German birth cohorts, GINI-plus and LISA-plus. Econ Hum Biol 2011;9:302-15.

52 Trasande L, Liu Y, Fryer G, et al. Effects of childhood obesity on hospital care and costs, 1999-2005. Health Aff 2009;28:w751-60.

53 Fleming-Dutra KE, Mao J, Leonard JC. Acute care costs in overweight children: a pediatric urban cohort study. Child Obes 2013:9:338-45.

54 Bechere Fernandes MT, Ferraro AA, Danti GV, et al. PP276-SUN: excess weight in children increases hospitalization days. Clinical Nutrition 2014;33:S122-3.

55 Kuhle S, Kirk S, Ohinmaa A, et al. Use and cost of health services among overweight and obese Canadian children. Int $J$ Pediatr Obes 2011;6:142-8.

56 Bianchi-Hayes J, Calixte R, Huang J, et al. Healthcare utilization by obese and overweight children. J Pediatr 2015;166:626-31.

57 Bettenhausen J, Puls H, Queen MA, et al. Childhood obesity and in-hospital asthma resource utilization. J Hosp Med 2015;10:160-4.

58 Doherty E, Queally M, Cullinan J, et al. The impact of childhood overweight and obesity on healthcare utilisation. Econ Hum Biol 2017;27:84-92

59 Dilley KJ, Martin LA, Sullivan C, et al. Identification of overweight status is associated with higher rates of screening for comorbidities of overweight in pediatric primary care practice. Pediatrics 2007;119:e148-55.

60 Reilly JJ, Kelly J, JJljoo K. Long-Term impact of overweight and obesity in childhood and adolescence on morbidity and premature mortality in adulthood: systematic review. Int $J$ Obes 2011;35:891-8

61 Wang F, McDonald T, Bender J, et al. Association of healthcare costs with per unit body mass index increase. J Occup Environ Med 2006;48:668-74

62 Wang YC, McPherson K, Marsh T, et al. Health and economic burden of the projected obesity trends in the USA and the UK. The Lancet 2011;378:815-25.

63 Pulgarón ER. Childhood obesity: a review of increased risk for physical and psychological comorbidities. Clin Ther 2013;35:A18-32.

64 Franklin J, Denyer G, Steinbeck KS, et al. Obesity and risk of low self-esteem: a statewide survey of Australian children. Pediatrics 2006:118:2481-7.

65 Strauss RS, Pollack HA, HAJAop P. Social marginalization of overweight children. Arch Pediatr Adolesc Med 2003;157:746-52.

66 Visness CM, London SJ, Daniels JL, et al. Association of childhood obesity with atopic and nonatopic asthma: results from the National Health and Nutrition Examination Survey 1999-2006. J Asthma 2010;47:822-9.

67 Cheung CRLH, Gray JAM, JMJAodic G. Unwarranted variation in health care for children and young people. Arch Dis Child 2013;98:60-5.

68 Finkelstein A, Gentzkow M, Williams H, HJTqjoe W. Sources of geographic variation in health care: evidence from patient migration. Q J Econ 2016;131:1681-726.

69 Godøy A, Huitfeldt I, IJJoHE H. Regional variation in health care utilization and mortality. J Health Econ 2020;71:102254.

70 World Health Organization. Report of the Commission on Ending Childhood Obesity: implementation plan: executive summary, 2017.

71 Caprio S, Daniels SR, Drewnowski A, et al. Influence of race, ethnicity, and culture on childhood obesity: implications for prevention and treatment: a consensus statement of Shaping America's Health and the Obesity Society. Diabetes Care 2008;31:2211-21.

72 Shrewsbury V, Wardle J. Socioeconomic status and adiposity in childhood: a systematic review of cross-sectional studies 19902005. Obesity

73 Coker TR, Elliott MN, Kataoka S, et al. Racial/ethnic disparities in the mental health care utilization of fifth grade children. Acad Pediatr 2009;9:89-96.

74 Fischer $\mathrm{AH}$, Shin DB, Margolis DJ, et al. Racial and ethnic differences in health care utilization for childhood eczema: an analysis of the 2001-2013 medical expenditure panel surveys. J Am Acad Dermato 2017;77:1060-7.

75 Amre DK, Infante-Rivard C, Gautrin D, et al. Socioeconomic status and utilization of health care services among asthmatic children. $J$ Asthma 2002:39:625-31.

76 Kangovi S, Barg FK, Carter T, et al. Understanding why patients of low socioeconomic status prefer hospitals over ambulatory care. Health Aff 2013;32:1196-203.

77 Szczepura A. Access to health care for ethnic minority populations. Postgrad Med J 2005;81:141-7. 
78 Scheppers E, van Dongen E, Dekker J, et al. Potential barriers to the use of health services among ethnic minorities: a review. Fam Pract 2006;23:325-48.

79 Kocken PL, Schönbeck Y, Henneman L, et al. Ethnic differences and parental beliefs are important for overweight prevention and management in children: a cross-sectional study in the Netherlands. BMC Public Health 2012:12:867.

80 Peña M-M, Dixon B, Taveras EM. Are you talking to ME? The importance of ethnicity and culture in childhood obesity prevention and management. Child Obes

81 Racine AD, Long TF, Helm ME, et al. Children's Health Insurance Program (CHIP): accomplishments, challenges, and policy recommendations. Pediatrics 2014;133:e784-93.
82 Dubay L, Kenney G. The impact of CHIP on children's insurance coverage: an analysis using the National Survey of America's Families. Health Serv Res 2009;44:2040-59.

83 Adams MH, Carter TM, Lammon CAB, et al. Obesity and blood pressure trends in rural adolescents over a decade. Pediatr Nurs 2008;34:381.

84 Bertoldi AD, Tavares NUL, Hallal PC, et al. Medicine use among adolescents: the 11-year follow-up of the 1993 Pelotas (Brazil) birth cohort study. Cad Saude Publica 2010;26:1945-53.

85 Wake M, Canterford L, Patton GC, et al. Comorbidities of overweight/ obesity experienced in adolescence: longitudinal study. Arch Dis Child 2010:95:162-8. 